\title{
Efficacy of Yushen Tongluo Granule Combined with Clomiphene Citrate for Anovulatory Infertility: A Double-Blind, Randomized, Placebo-Controlled Clinical Trial
}

\author{
Huayun Xu $\mathbb{D},{ }^{1}$ Songpo Wang, ${ }^{1}$ Xiaohong Gao, ${ }^{2}$ and Guozeng Wang $\mathbb{D}^{3}$ \\ ${ }^{1}$ Department of Traditional Chinese Medicine, Shanghai General Hospital, Shanghai Jiao Tong University School of Medicine, \\ Shanghai 200080, China \\ ${ }^{2}$ Department of Obstetrics and Gynecology, Shanghai General Hospital, Shanghai Jiao Tong University School of Medicine, \\ Shanghai 200080, China \\ ${ }^{3}$ Department of Obstetrics and Gynecology, Shanghai East Hospital, Tongji University School of Medicine, \\ Shanghai 200120, China
}

Correspondence should be addressed to Guozeng Wang; zengerwong@126.com

Received 6 October 2021; Revised 4 January 2022; Accepted 8 January 2022; Published 27 January 2022

Academic Editor: Samuel Martins Silvestre

Copyright (c) 2022 Huayun Xu et al. This is an open access article distributed under the Creative Commons Attribution License, which permits unrestricted use, distribution, and reproduction in any medium, provided the original work is properly cited.

Objective. To evaluate the efficacy and safety of Yushen Tongluo Granule (YSTLG) combined with clomiphene citrate (CC) in the treatment of anovulatory infertility. Methods. This randomized, double-blinded, placebo-controlled clinical trial was carried out in the Department of Obstetrics and Gynecology and the Department of Traditional Chinese Medicine (TCM). During the 3 menstrual cycle intervention periods, all subjects received $50 \mathrm{mg} /$ day CC from day 5 until day 9 of the menstruation. If no ovulation, the amount of CC per cycle increased $50 \mathrm{mg}$ /day until $150 \mathrm{mg} /$ day. Participants in the experimental group received YSTLG, while participants in the control group received YSTLG placebo. The granules were orally taken from the end of menstruation until ovulation. When one leading follicle attained a diameter of $18 \mathrm{~mm}$ or more, $5000 \mathrm{U}$ human chorionic gonadotropin (hCG) was given intramuscularly. The primary outcome measure was the ovulation rate, and follicular development was monitored by transvaginal ultrasound on the $10^{\text {th }}$ day of the cycles until ovulation. Secondary outcome measures including the overall curative effect, endometrial thickness, and pregnancy outcomes were also compared between the two groups. Results. The ovulation rate in the experimental group was higher than that in the control group $(P<0.05)$. Compared with the control group, the overall curative effect of the experimental group was better than that of the control group $(P<0.05)$, and the endometrial thickness in the ovulation phase was significantly thicker than that in the control group $(P<0.01)$. There was no significant difference in pregnancy rate and miscarriage rate between the experimental group and control group $(P>0.05)$. Conclusion. The combined YSTLG and CC used to treat anovulatory infertility can improve the ovulation rate without affecting endometrial thickness, which is efficacious and safe.

\section{Introduction}

Infertility is a group of reproductive disorders caused by a variety of causes. In China, $25 \%$ of couples actively attempting to become pregnant suffered infertility [1]. Infertility is a heavy burden on the family, especially for women, which can often cause severe anxiety and depression $[2,3]$. Anovulation is an important cause of female infertility. It is found that $85 \%$ of anovulation was led to hypothalamic-pituitary-ovarian axis disorders caused by polycystic ovary syndrome (PCOS), abnormal body mass index (BMI), and endocrine diseases [4]. Therefore, restoring ovulation is an important link in the treatment of infertility.

As a nonsteroidal selective estrogen receptor regulator, CC, the first-line ovulation promoting drug, interferes with estrogen receptor in hypothalamus and blocks the negative feedback of estrogen on gonadotropin secretion, thus promoting follicular development [5]. However, due to its peripheral antiestrogen effect, CC is investigated to induce endometrial dysplasia and affect embryo implantation $[6,7]$. Therefore, a novel therapy promoting 
ovulation without affecting the growth and development of endometrium is significant.

Traditional Chinese medicine has a long history in the treatment of infertility, which guaranteed a reliable outcome. The clinical data showed that traditional Chinese medicine had obvious characteristics and advantages in the treatment of ovulation induction on female infertility [8]. In addition, research studies also demonstrated that the performance of traditional Chinese medicine could regulate sex hormones to promote follicular development and improve endometrial receptivity in the treatment of ovulation disorder infertility $[9,10]$. At the same time, some studies also find that Chinese herbal medicine may increase the effectiveness of CC therapy [11].

YSTLG contains pure, natural herbal ingredients; it is an empirical prescription developed by Professor Xiaosun Cai, a famous TCM physician. Until now, it has been successfully used in the clinical treatment for more than 30 years and has a significant therapeutic effect on anovulatory infertility. We speculated that YSTLG combined with CC in the treatment of anovulatory infertility may improve the ovulation rate without affecting the endometrial thickness during ovulation, so as to increase the pregnancy rate and reduce the abortion rate. Therefore, the goal of this trial was to evaluate the efficacy and safety of YSTLG combined with CC in the treatment of anovulatory infertility in a randomized, doubleblind, placebo-controlled clinical trial.

\section{Methods}

2.1. Study Design. The study was a 3 menstrual cycle, doubleblind, randomized, placebo-controlled clinical trial, conducted in the Department of Obstetrics and Gynecology and the Department of TCM of Shanghai General Hospital from August 2016 to July 2018.

This study was approved by the Ethics Committees of Shanghai General Hospital (grant no. (2017)09). Also, it was registered in the Chinese Clinical Trial Registry with the code number of ChiCTR2000040753 (https://www.chictr. org.cn/historyversionpuben.aspx?

regno=ChiCTR2000040753). The study was carried out in accordance with the Declaration of Helsinki, and all eligible participants provided hand-written informed consent.

2.2. Drug Preparation. CC was obtained from Codal Synto Limited. YSTLG (12 g/bag) and YSTLG placebo (composed of $10 \%$ crude drug of YSTLG, dextrin, and stevia, $12 \mathrm{~g} / \mathrm{bag}$ ) were produced by the Shanghai Liantang Pharmaceutical Limited Company. The recipe in YSTLG consisted of Yinyanghuo (Herba Epimedii), Guizhi (Ramulus Cinnamomi), Bajitian (Radix Morindae Officinalis), Maidong (Radix Ophiopogonis), Dihuang (Radix Rehmanniae), Shudihuang (Radix Rehmanniae Preparata), Lulutong (Fructus Liquidambaris), Dingxiang (Flos Caryophylli), Shinanye (Folium Photiniae), and Huangjing (Rhizoma Polygonati). HCG was obtained from Shanghai First Biochemical Pharmaceutical Limited Company.

\subsection{Diagnostic Criteria}

2.3.1. Diagnostic Criteria for Western Medicine. According to obstetrics and gynecology [12], Guiding Principles of Clinical Research on New Drugs of Traditional Chinese Medicine [13], the diagnostic criteria for anovulatory infertility are as follows: the inability to conceive after 1 year of unprotected intercourse of reasonable frequency, basal body temperature (BBT) charts showed no biphasic pattern for more than 3 months, vaginal smear showed no periodic change, cervical mucus crystallization showed no ellipsoid appearance, endometrial biopsy within 6 days of cycle showed no typical secretory phase change, and the level of progesterone was lower than that in luteal-phase. A diagnosis of anovulatory was made based on (1) and at least two of (2)-(6) were met.

\subsubsection{Criteria of TCM Differentiation of Symptoms and Signs.} Kidney essence insufficiency pattern was diagnosed based on the Routine Diagnosis and Treatment of Traditional Chinese Medicine Syndrome in Shanghai [14] and National Standard for Clinical Terms of Traditional Chinese Medicine [15]. Primary symptom patterns included infertility following regular marital life for at least 1 year, delayed menstrual cycle and oligohypomenorrhea or amenorrhea, soreness, and weakness of waist and knees. Secondary symptom patterns included dizziness and tinnitus, sexual hypoactivity, amnesia, alopecia, fatigue, stringy, or deep and faint pulse. Diagnosis was made if 3 primary symptom patterns plus 2 secondary symptom patterns were identified.

2.4. Inclusion and Exclusion Criteria. Participants were adults aged 20-40 years old, who corresponded to the diagnosis criteria of anovulatory infertility and TCM syndrome differentiation. Eligible patients voluntarily joined the clinical observation and signed informed consent.

Exclusion criteria were as follows: infertility caused by congenital genitalia defects or malformations, infertility caused by genetic factors, infertility caused by endometriosis, adenomyosis, leiomyomas, and uterine dysplasia, infertility caused by tubal obstruction, immune infertility, infertility caused by male reproductive dysfunction, use of hormonal drugs (except for progesterone) or any other drugs which can affect reproductive endocrine in the past 3 months, complicated with severe cardiac, pulmonary, hepatic, renal, or neurological disease or mental illness, and complicated with thrombotic diseases.

2.5. Randomization and Blinding. Eligible participants were randomly assigned to groups $\mathrm{A}$ and $\mathrm{B}$ at a ratio of $1: 1$ according to the random number table. The table was generated through SPSS 18.0. Group A and group B corresponded to the experimental group (YSTLG) or control group (YSTLG placebo), respectively. YSTLG and YSTLG placebo had the same appearance, shape, color, and packaging, and the research physicians and participants were not able to know the difference. 
2.6. Intervention. Both the experimental group and control group were given $50 \mathrm{mg} /$ day CC for 5 days on the 5 th day after the onset of spontaneous and progestin-induced menstruation as a basic treatment. If no ovulation, the amount of CC per cycle increased $50 \mathrm{mg}$ /day until $150 \mathrm{mg} /$ day. During the trial, participants in the experimental group were treated with YSTLG (12 g/bag, one bag per time, twice a day), while those in the control group were treated with YSTLG placebo (12 g/bag, one bag per time, twice a day). The granules were orally taken from the end of menstruation until ovulation. When one leading follicle attained a diameter of $18 \mathrm{~mm}$ or more, $5000 \mathrm{U}$ hCG intramuscular injection was given. Timed intercourse was advised starting every other day for 1 week from the night of hCG administration. The treatment lasted for a total of 3 menstrual cycles, and drug administration was discontinued once the participant became pregnant during the trial.

2.7. Efficacy Criteria. According to the reference, clinical control (I): most of the clinical symptoms disappeared and the TCM syndrome total score decreased no less than 95\%; significantly effective (II): most of the clinical symptoms disappeared and the TCM syndrome total score decreased no less than 70\%; effective (III): some of the clinical symptoms improved and the TCM syndrome total score decreased no less than 30\%; ineffective (IV): clinical symptoms did not improve or even deteriorate and the TCM syndrome total score decreased less than 30\% [16].

\subsection{Outcomes}

2.8.1. Primary Outcomes. Follicular development monitored by transvaginal ultrasound: transvaginal follicular monitoring was performed on all participants on days 10 and then individualized according to response. The ultrasound was performed once in 3 days when the diameter of the dominant follicle was less than $10 \mathrm{~mm}$, every other day when $10-15 \mathrm{~mm}$, and every day when more than $15 \mathrm{~mm}$. Meanwhile, the maximum diameter of the dominant follicle during ovulation was recorded.

2.8.2. Secondary Outcomes. (1) Endometrial lining monitored by transvaginal ultrasound: when one leading follicle attained a diameter of $15 \mathrm{~mm}$ or more, the endometrial thickness was recorded daily till ovulation by transvaginal endometrial lining monitoring. (2) Pregnant rate and miscarriage rate: a serum $\beta$-hCG concentration was determined 14 days after hCG injection if menses had not yet occurred. Biochemical pregnancy was defined as a falling $\beta$-hCG concentration on serial determination. Clinical pregnancy was diagnosed by visualization of a gestational sac with fetal heartbeat. Miscarriages were defined as biochemical pregnancies and/or cases with positive $\beta$-hCG testing who aborted spontaneously before reaching the stage of clinical pregnancy and/or cases aborting before 12 weeks of pregnancy.
2.9. Safety. General medical examination and regular blood tests, including blood routine and glutamic-pyruvic transaminase, glutamic-oxaloacetic transaminase, blood urea nitrogen, and creatinine, were examined before and after the trial treatment. All adverse events were recorded in the case report during the clinical trial. Additionally, the participants were provided with the researcher's telephone number and were instructed to call the researcher in case of possible side effects or deterioration of health.

2.10. Statistical Analysis. Statistical analysis was performed with the Statistical Package for the Social Science (SPSS Inc., Chicago, IL, USA) version 18.0. Quantitative data were summarized as mean \pm standard deviation $(\bar{x} \pm s)$. According to the normal distribution, the paired $t$-test was used for before and after treatment comparisons within the treatment and control groups, while the independent samples $t$-test was used between the two groups. To those who did not conform to normal distribution, the nonparametric test of rank transformation was used. Count data used case or percentage representation. The chi-square test was used for nonrank data and rank sum test for rank data. $P<0.05$ was considered statistically significant.

\section{Results}

3.1. Patients Enrollment. As shown in Figure 1, of the 100 patients assessed for participation in the study, 5 did not meet the inclusion criteria and 3 refused to participate. A total of 92 recruited patients were allocated to each group (each group comprising 46 patients). During the study period, 4 of the 92 patients were lost. Of the 4 lost, 2 were from the experimental group and 2 were from the control group. Analysis included 43 patients in the experimental group and 43 patients in the control group.

\subsection{Comparison of Baseline Characteristics between the Two} Groups. Baseline demographic characteristics of all participants are given in Table 1. There was no significant difference in age, infertility duration, BMI, the number of PCOS, and reproductive history between the two groups $(P>0.05)$.

3.3. Primary Outcome Measures. As given in Table 2, the ovulation rate of the experimental group was higher than that of the control group, and the difference was statistically significant $(P<0.05)$.

\subsection{Secondary Outcome Measures}

3.4.1. Comparison of Clinical Efficacy between the Two Groups after Treatment. As given in Table 3, the number of cured cases in the experimental group and the control group was 30 cases and 21 cases, respectively. Among them, 25 cases were pregnant in the experimental group and 21 cases in the control group. There was a significant difference in the overall efficacy between the two groups after treatment $(P<0.05)$. 


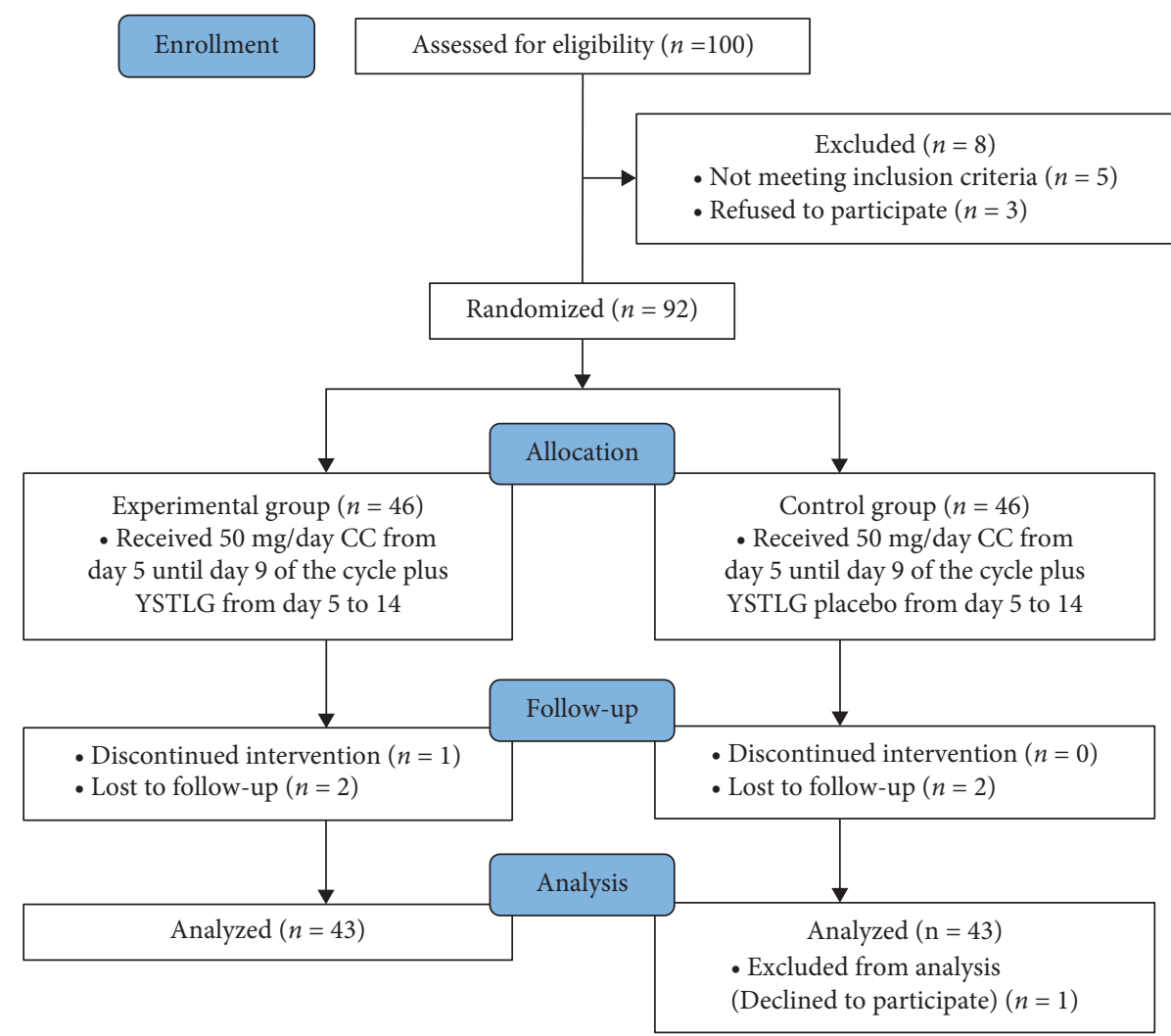

FIgURE 1: Flow diagram of patient enrollment, allocation, follow-up, and analysis.

TABLE 1: Baseline demographic characteristics of study participants.

\begin{tabular}{|c|c|c|c|}
\hline Characteristics & Experimental group $(n=43)$ & Control group $(n=43)$ & $P$ value \\
\hline Age $(\bar{x} \pm s$, years $)$ & $30.67 \pm 4.50$ & $31.19 \pm 4.58$ & $0.603^{*}$ \\
\hline Infertility duration $(\bar{x} \pm s$, years $)$ & $2.26 \pm 1.02$ & $2.50 \pm 0.99$ & $0.264^{*}$ \\
\hline BMI $(\bar{x} \pm s$, years $)$ & $22.52 \pm 1.36$ & $22.83 \pm 1.33$ & $0.288^{*}$ \\
\hline $\operatorname{PCOS}(n(\%))$ & $31(72.09)$ & $31(72.09)$ & - \\
\hline Reproductive history & - & - & - \\
\hline Childbirth $(n(\%))$ & $8(18.60)$ & $7(16.28)$ & $0.776^{* *}$ \\
\hline Spontaneous abortion $(n(\%))$ & $18(41.86)$ & $12(27.91)$ & $0.175^{* *}$ \\
\hline
\end{tabular}

${ }^{*}$ Independent $t$-test. ${ }^{* *}$ Chi-square test.

TABLE 2: Comparison of ovulation rate between the two groups.

\begin{tabular}{lccc}
\hline Group & $n$ & Treatment cycles & Ovulation cycles $(n(\%))$ \\
\hline Experimental & 43 & 91 & $82(90.10)$ \\
Control & 43 & 99 & $78(78.79)$ \\
$P$ value & - & - & 0.033 \\
\hline
\end{tabular}

Values are reported as $n(\%)$ and analyzed by the chi-square test. Ovulation rate $=$ ovulation cycles/treatment cycles $\times 100 \%$.

TABle 3: Comparison of clinical efficacy $(n(\%))$.

\begin{tabular}{llccccc}
\hline Group & $n$ & I & II & III & IV & Total effective, \% \\
\hline Experimental & 43 & $30(69.8)$ & $5(11.6)$ & $6(14.0)$ & $2(4.7)$ & 95.3 \\
Control & 43 & $21(48.8)$ & $2(4.7)$ & $17(39.5)$ & $3(7.0)$ & 93.0 \\
\hline
\end{tabular}

Analyzed by the nonparametric rank sum test, $Z=2.237, P=0.025$. I, clinical control; II, significantly effective; III, effective; IV, ineffective. 
TABle 4: Comparison of endometrial thickness during ovulation between the two groups $(\bar{x} \pm s)$.

\begin{tabular}{lccc}
\hline Group & Endometrial thickness $\left(1^{\text {st }}\right.$ cycle $)(\mathrm{mm})$ & Endometrial thickness $\left(2^{\text {nd }}\right.$ cycle $)(\mathrm{mm})$ & Endometrial thickness $\left(3^{\text {rd }}\right.$ cycle $)(\mathrm{mm})$ \\
\hline Experimental & $9.73 \pm 2.11$ & $9.38 \pm 2.10$ & $9.43 \pm 2.29$ \\
Control & $7.80 \pm 2.25$ & $7.22 \pm 1.40$ & $7.81 \pm 1.72$ \\
$P$ value & $<0.001$ & $<0.001$ & 0.007 \\
\hline
\end{tabular}

TABLE 5: Comparison of pregnancy rate and abortion rate between the two groups.

\begin{tabular}{|c|c|c|c|c|c|}
\hline Group & $\begin{array}{c}\text { Pregnancy cases }\left(1^{\text {st }}\right. \\
\text { cycle })\end{array}$ & $\begin{array}{c}\text { Pregnancy cases }\left(2^{\text {nd }}\right. \\
\text { cycle })\end{array}$ & $\begin{array}{c}\text { Pregnancy cases }\left(3^{\text {rd }}\right. \\
\text { cycle })\end{array}$ & $\begin{array}{c}\text { Total number of } \\
\text { pregnancies }\end{array}$ & Abortions \\
\hline Experimental & 17 & 4 & 4 & $25(58.1)$ & $1(4.0)$ \\
\hline Control & 14 & 2 & 5 & $21(48.8)$ & $2(9.5)$ \\
\hline$P$ value & - & - & - & 0.387 & 0.585 \\
\hline
\end{tabular}

Values are reported as $n(\%)$ and analyzed by the chi-square test.

3.4.2. Comparison of Endometrial Thickness during Ovulation between the Two Groups. As given in Table 4, for nonpregnant women, endometrial thickness was monitored by continuous transvaginal ultrasound. The results showed that endometrial thickness increased significantly during the first, second, and third ovulation periods in the experimental group compared with the control group $(P<0.01)$.

3.4.3. Comparison of Pregnancy Rate and Abortion Rate between the Two Groups. As given in Table 5, after treatment, more than half of the patients in the experimental group were pregnant, of which only 1 was aborted. There were 21 cases of pregnancy and 2 cases of abortion in the control group. There was no significant difference between the experimental group and the control group $(P>0.05)$.

3.5. Adverse Reactions. There were no adverse reactions observed in either group during the trial treatment period.

\section{Discussion}

One major strength is that this study is a randomized, double-blind, placebo-controlled clinical trial. For clinical trials, placebo-controlled trials can reliably demonstrate the efficacy of research drugs and can distinguish whether adverse events are caused by drugs. After 3 menstrual cycles of intervention, in this clinical trial, as ovulation rate was our primary outcome, the overall efficacy of the experimental group was better than that of the control group. Furthermore, the endometrium of 3 menstrual cycles ovulation of the experimental group was significantly thicker than that of the control group. We can conclude that the combined therapy with YSTLG and CC can improve ovulation rate and cannot affect the endometrial thickness during ovulation. Both of these are preconditions for a successful clinical pregnancy.

In addition, the number of cured and significantly effective cases in the experimental group is more than the control group, and there was a significant difference in the overall efficacy between the two groups after treatment. It showed that the overall efficacy of the combined therapy with YSTLG and CC was better than CC alone. However, there was no significant difference in the pregnancy rate and miscarriage rate between two groups $(P>0.05)$. Therefore, now, there was no evidence that the combined therapy can improve the pregnancy rate and reduce the abortion rate.

YSTLG has the effect of nourishing kidney and filling essence, promoting blood circulation, and dredging collaterals. Some studies found that the traditional Chinese medicine could increase the level of estradiol (E2) [17] and promote the blood supply of endometrium, induce angiogenesis, and improve endometrial receptivity significantly [18]. Besides, the drugs for promoting blood circulation and removing blood stasis can promote the proliferation of ovarian granulosa cells [19] and improve the blood supply to the uterus, resulting in increased blood flow of endometrium, hence promoting the growth and development of decidua [20]. A growing body of evidence supports that some herbal ingredients of YSTLG have estrogen-like effects, improve ovarian endocrine function, or promote angiogenesis. For example, it is found that estrogen-like substances in Herba Epimedii, which significantly increase the serum level of E2 [21], and icariin, an extract from Herba Epimedii, can thick the endometrium by increasing the expression levels of ER, VEGF, and KDR in endometrial cells [22] and improve the ovarian endocrine function [23]. Pharmacological studies show that Radix Rehmanniae Preparata has phytoestrogenic effects and could increase the immature rat's uterus wet weight and the ratio of uterus to body weight [24], and catalpol, an extract from Radix Rehmanniae Preparata, can improve both the quality and quantity of follicles on the sex organs [25]. It is also found that inulin-type oligosaccharides from Radix Morindae Officinalis roots promote angiogenesis by activating the PI3K-PKB-eNOS-signaling pathway [26]. Besides, Ramulus Cinnamomi and its active compounds can induce angiogenesis by upregulating the expression of VEGF in endothelial cells [27]. Therefore, we speculate that YSTLG may improve the ovulation rate and endometrial thickness by increasing the estrogen level or promoting endometrial angiogenesis, but the exact molecular mechanism remains unclear and requires further investigation.

This is the first time that a combination of YSTLG and CC has been studied for the treatment of anovulatory infertility. To date, only some studies have assessed the efficacy 
of traditional Chinese prescriptions for treating anovulation, and the quality of the evidence for other comparisons and outcomes was very low [28]. Similarly, nowadays, there is only limited evidence to suggest that the addition of Chinese herbal medicine to $\mathrm{CC}$ may improve pregnancy rates, but the quality of the evidence is low [28]. Our trial did not find that there were any significant differences between the experimental group and the control group in terms of pregnancy rate and miscarriage rate, which may be related to the small sample size, short intervention duration, and no postintervention follow-up. Moreover, in terms of safety, there were no adverse reactions observed between two groups during the trial treatment period.

In conclusion, the present study suggests that the combined treatment of YSTLG and CC used to treat anovulatory infertility is efficacious and safe, which can improve the ovulation rate without affecting endometrial thickness during ovulation, and make up for the defect of CC. The overall clinical effect of the combination therapy was better than that of CC alone. However, due to the small sample size, a confirmative conclusion is still premature. These findings could be used as preliminary data for further large-scale studies.

\section{Data Availability}

The data used to support the findings of this study are available from the corresponding author upon request.

\section{Ethical Approval}

The study protocol was confirmed by the Ethics Committees of Shanghai General Hospital (grant no. (2017)09) and followed the Declaration of Helsinki and its subsequent revisions.

\section{Consent}

Written informed consent was signed before participation in the trial.

\section{Disclosure}

The funding sources were not involved in the study design, collection, analysis, and interpretation of data, or the decision to submit the manuscript for publication.

\section{Conflicts of Interest}

The authors declare that they have no conflicts of interest.

\section{Authors' Contributions}

Huayun Xu and Guozeng Wang contributed to study design, data acquisition, data analysis, and manuscript draft. Songpo Wang and Xiaohong Gao contributed to data collection. All authors read and approved the final version.

\section{Acknowledgments}

The authors thank all the patients who participated in this study and all the medical staff in the Department of TCM and the Department of Obstetrics and Gynecology, Shanghai General Hospital, for their contributions to this article. This research was supported by the Special Project of Traditional Chinese Medicine Scientific Research of Shanghai Health and Family Planning Commission (2016LP024) and "Xinglin Xinxing" Talent Project of Shanghai ( ZY (2018-2020)RCPY-3006).

\section{References}

[1] Z. Zhou, D. Zheng, H. Wu et al., "Epidemiology of infertility in China: a population-based study," BJOG: An International Journal of Obstetrics and Gynaecology, vol. 125, no. 4, pp. 432-441, 2018.

[2] K. L. Rooney and A. D. Domar, "The relationship between stress and infertility," Dialogues in Clinical Neuroscience, vol. 20, pp. 41-47, 2018.

[3] A. Pozza, D. Dèttore, and M. E. Coccia, "Depression and anxiety in pathways of medically assisted reproduction: the role of infertility stress dimensions," Clinical Practice \& Epidemiology in Mental Health, vol. 15, pp. 101-109, 2019.

[4] S. Mikhael, A. P. Patel, and L. G. Jordan, "Hypothalamicpituitary-ovarian Axis disorders impacting female fertility," Biomedicines, vol. 7, no. 1, 2019.

[5] J. Yun, Y. S. Choi, I. Lee et al., "Comparison of congenital malformations among babies born after administration of letrozole or clomiphene citrate for infertility treatment in a Korean cohort," Reproductive Toxicology, vol. 82, pp. 88-93, 2018.

[6] M. F. Mitwally and R. Casper, "The aromatase inhibitor, letrozole: a promising alternative for clomiphene citrate for induction of ovulation," Fertility and Sterility, vol. 74, 2000.

[7] I. Wagman, I. Levin, and R. Kapustiansky, "Clomiphene citrate vs. letrozole for cryopreserved-thawed embryo transfer: a randomized, controlled trial," Journal of Reproductive Medicine, Gynaecology, and Obstetrics, vol. 55, pp. 134-138, 2010.

[8] L. Chen, Y. Tan, and S. P. Chen, "Effect of clomiphene citrate and Dingkun Dan on ovulation induction and clinical pregnancy of polycystic ovary syndrome," Zhongguo Zhong Yao Za Zhi, vol. 42, pp. 4035-4039, 2017.

[9] J. Feng Xia, Y. Inagaki, J. Feng Zhang, L. Wang, and P. Pei Song, "Chinese medicine as complementary therapy for female infertility," Chinese Journal of Integrative Medicine, vol. 23, pp. 245-252, 2017.

[10] M. Xin, J. He, W. Yang, X. Yin, and J. Wang, "Wenshen Yangxue decoction improves endometrial receptivity recovery and promotes endometrial angiogenesis in a rat model," Pharmaceutical Biology, vol. 56, no. 1, pp. 573-579, 2018.

[11] C. See, M. Mcculloch, and C. Smikle, "Chinese herbal medicine and clomiphene citrate for anovulation: a metaanalysis of randomized controlled trials," Journal of Alternative and Complementary Medicine, vol. 17, pp. 397-405, 2011.

[12] X. Xie, B. Kong, and T. Duan, Infertility in Obstetrics and Gynecology, pp. 361-363, People's Health Publishing House, Beijing, China, 2018.

[13] Ministry of Health of the People's Republic of China, Guiding Principles of Clinical Research on The Treatment of Female 
Infertility by New Chinese Medicine, pp. 276-280, Ministry of health of the People's Republic of China, Beijing, China, 1993.

[14] G. Liu and M. Zhang, Routine Diagnosis and Treatment of Traditional Chinese Medicine Syndrome in Shanghai, Shanghai University of Chinese Medicine Press, Shanghai, China, 2003.

[15] State Bureau of Technology Supervision, "National Standard for Clinical Terms of Traditional Chinese Medicine: GB/T 16751.2-1997, China Standard Press, Beijing, China, 1997.

[16] State Administration of Traditional Chinese Medicine, Criteria for the Diagnosis and Treatment of Traditional Chinese Medicine Syndrome, Nanjing University Press, Nanjing, China, 1994.

[17] H. F. Li, Q. H. Shen, W. J. Chen, and W. M. Chen, "Efficacy of traditional Chinese medicine tonifying kidney (bushen) and activating blood (huoxue) prescription for premature ovarian insufficiency: a systematic review and meta-analysis," Evidence-Based Complementary and Alternative Medicine, vol. 2020, Article ID 1789304, 13 pages, 2020.

[18] D.-m. Huang, G.-y. Huang, and F.-e. Lu, "Effects of bushenyiqihexue formula on the endometrial gland apoptosis in mice with blastocyst implantation dysfunction," Journal of Traditional Chinese Medicine, vol. 30, no. 3, pp. 196-200, 2010.

[19] D. Yao, Z. Wang, L. Miao, and L. Wang, "Effects of extracts and isolated compounds from safflower on some index of promoting blood circulation and regulating menstruation," Journal of Ethnopharmacology, vol. 191, pp. 264-272, 2016.

[20] S. T. Huang and A. P. C. Chen, "Traditional Chinese medicine and infertility," Current Opinion in Obstetrics and Gynecology, vol. 20, no. 3, pp. 211-215, 2008.

[21] F.-F. Yan, Y. Liu, Y.-F. Liu, and Y.-X. Zhao, "Herba Epimedii water extract elevates estrogen level and improves lipid metabolism in postmenopausal women," Phytotherapy Research, vol. 22, no. 9, pp. 1224-1228, 2008.

[22] A. W. Le, L. Shan, Z. H. Wang, X. Y. Dai, T. H. Xiao, and R. Zuo, "Effects of icariin on the expression of ER, VEGF, and KDR in the endometrial cells of thin endometrium," Genetics and Molecular Research, vol. 14, no. 3, pp. 11250-11258, 2015.

[23] X. Nie, W. Sheng, D. Hou, Q. Liu, R. Wang, and Y. Tan, "Effect of Hyperin and Icariin on steroid hormone secretion in rat ovarian granulosa cells," Clinica Chimica Acta, vol. 495, pp. 646-651, 2019.

[24] Q. Hao, J. Wang, and J. Niu, "Study on phytoestrogenic-like effects of four kinds of Chinese medicine including Radix Rehmanniae preparata, Radix paeoniae alba, Radix angelicae sinensis, rhizoma chuanxiong," Zhongguo Zhongyao Zazhi, vol. 34, pp. 620-624, 2009.

[25] M. Wei, Y. Lu, D. Liu, and W. Ru, "Ovarian failure-resistant effects of catalpol in aged female rats," Biological and Pharmaceutical Bulletin, vol. 37, no. 9, pp. 1444-1449, 2014.

[26] X. Cha, S. Han, J. Yu et al., "Inulin with a low degree of polymerization protects human umbilical vein endothelial cells from hypoxia/reoxygenation-induced injury," Carbohydrate Polymers, vol. 216, pp. 97-106, 2019.

[27] D. Y. Choi, Y. H. Baek, and J. E. Huh, "'Stimulatory effect of Cinnamomum cassia and cinnamic acid on angiogenesis through up-regulation of VEGF and Flk-1/KDR expression," Immunopharmacology, vol. 9, pp. 959-967, 2009.

[28] M. Ong, J. Peng, X. Jin, and X. Qu, "Chinese herbal medicine for the optimal management of polycystic ovary syndrome," The American Journal of Chinese Medicine, vol. 45, no. 03, pp. 405-422, 2017. 